\title{
Range assignment problem on the Steiner tree based topology in ad hoc wireless networks
}

\author{
Rashid Bin Muhammad \\ Department of Computer Science, Kent State University, Kent, OH, USA \\ Tel.: +1 330 6722074; Fax: +1 330672 0737;E-mail: rmuhamma@cs.kent.edu
}

\begin{abstract}
This paper describes an efficient method for introducing relay nodes in the given communication graph. Our algorithm assigns transmitting ranges to the nodes such that the cost of range assignment function is minimal over all connecting range assignments in the graph. The main contribution of this paper is the $O(N \log N)$ algorithm to add relay nodes to the wireless communication network and 2-approximation to assign transmitting ranges to nodes (original and relay). It does not assume that communication graph to be a unit disk graph. The output of the algorithm is the minimal Steiner tree on the graph consists of terminal (original) nodes and relay (additional) nodes. The output of approximation is the range assignments to the nodes.
\end{abstract}

Keywords: Connectivity, range assignment, steiner tree, and approximation

\section{Introduction}

An ad hoc wireless network is a collection of independent devices (transceiver nodes) that have to communicate themselves in the absence of any central authority. The absence of central authority implies that the coordination necessary for communication has to be carried out by the nodes themselves. Ad hoc network nodes allow to communicate directly to each other using wireless transceivers without need for a fixed infrastructure. Any two devices (nodes) in the region achieve communication either directly if they are within each others transmission range (i.e., within one hop) or indirectly via other nodes, if they are out of each others range (multi-hop). Note that in this work we assume that transceiver nodes are portable devices. Typically, portable devices have limited power resource available. One of the main characteristic properties of the ad hoc wireless networks is the ability to vary the power used in communication among nodes, which implies the concept of transmission range, hence the range assignment.

There are many situations in which fixed wired networks, such as the Internet, are not realistic or practicable because it may not physically possible (due to fire, earthquake or flood) to deploy wired network or due to economic infeasibility. In such situations a collection of hosts (emergency workers) with wireless devices may form a temporary network (ad hoc wireless network). Furthermore, it is not hard to imagine that soon after the initial deployment of emergency network, we need to expand this network by adding additional hosts (additional emergency workers). Now the question is where we deploy these additional hosts so that the final network is connected and use minimal communication 
power. The motivation for our problem arises from this very scenario [19]. Consider a group of emergency workers, e.g. fire fighters, deployed in some geographical region. These workers need to communicate by transmitting and receiving radio signals on an ad hoc network. Workers are equipped with terminal devices. From now on, we call them terminal nodes. A support team with additional transceivers, called relay nodes, are use to form the connected network. The task of the support team is to place relay nodes in the region so that the terminal nodes and the relay nodes together can form a connected communication graph while at the same time minimizing the energy consumption by setting transmitting ranges. Note that the preliminary version of this work appeared in Reference [23].

A range assignment for the finite set $N$ of $n$ network nodes is a function, $R A: \rightarrow R^{+}$(where $R^{+}$is the set of non-negative numbers), that assigns to every node $u \in N$ such that $0<R A(u) \leqslant r_{\max }$, where $r_{\max }$ is the maximum transmitting range. The cost of a range Assignment is defined as $\operatorname{cost}(R A)=$ $\Sigma(R A(u))^{\alpha}$ for some real constant $\alpha>0$. The underlying intuition is that the element of a finite set $N$ are given transceiver nodes, and one can choose for each transceiver nodes $u \in N$ a corresponding data transmission range i.e., radius, $R A(u)$. Sending data at radius, $R A(u)$, consumes energy proportional to $(R A(u))^{\alpha}$. The parameter is $\alpha$ called the distance-power gradient. In an ideal environment, i.e., in the empty space $\alpha=2$ but vary from 1 to more than 6 depending on the environmental conditions of the place where the transceiver nodes are located, see [25].

Let $\operatorname{dist}(u, v)$ denote the Euclidean distance between two arbitrary nodes $u, v \in R^{2}$. We define range assignment $R A$ on the following kind of graph. Let $G_{R A}=\left(N, E_{R A}\right)$ be directed communication graph of range assignment $R A$ where an edge (communication link) belongs to the set $E_{R A}$ if, and only if, node $u$ can send the data to node $u$, i.e. the radius of node $u$ is at least as large as the distance between nodes $u$ and $v$. Formally, we say as follows:

Proposition 1. Given nodes $u, v \in\left(N, E_{R A}\right)$, edge $(u, v) \leqslant E_{R A}$ if, and only if, $R A(u) \geqslant \operatorname{dist}(u, v)$.

In this work, we demand that the induced communication graph $G_{R A}$ must be strongly connected to ensure all-to-all communication. Note that here by strong connectivity we mean the relevant optimization problem, i.e. the problem to find a range assignment $R A$ that has minimal $\operatorname{cost}(R A)$ among all range assignments satisfying the strong connectivity property.

Prosopition 2. Given directed communication graph $G_{R A}$ is strongly connected if every two node $u, v \in G_{R A}$ can send data to each other i.e. $u$ and $v$ are reachable from each other.

The paper is organized as follows. In the next section we define the problem. In the Section 3 we discuss the relevant work. Preliminaries and definition are given in Section 4. Section 5 presents the computational model. Section 6 describes the algorithm based on the Steiner tree. Section 7 presents 2-Approximation algorithm for range assignment problem. Section 8 discuss the open problem. Finally, Section 9 concludes the paper with some final remarks.

\section{Problem definition}

We shall formulate the problem in the geometric graph, using [15,20], as follows. Given a set $N$ of $n$ points, the problem is to introduce minimal number of additional points such that the graph $G\left(N \cup N_{R A}, R A\right)$ is strongly connected and the cost of range assignment function, $\operatorname{Cost}(R A)=$ $\sum(R A(u))^{\alpha}$ is minimum over all connecting range assignment functions, where $\alpha$ is the distance-power gradient. Note that if range assignment function $R A=0$ (i.e. transmitting range $r=0$ ), this problem 
reduces to finding the Euclidean Steiner tree problem [21] for the set $N$ and in context of ad hoc wireless networking, the solution of this problem is proposed in [20,22].

Given the location of the terminal nodes, we are interested in the problem of establishing strong connectivity by minimizing the number points (physical loations) where the support team should placed additional relay nodes and minimizing the range assignment, $R A$, of the resultant graph (tree). In a certain sense, this problem can be seen as a generalization of the problem of determining the critical transmitting range for connectivity, where the constraint that all the nodes have the same transmitting range is dropped. Note that the solution of the problem in which given nodes have the same transmitting range is proposed in [15,19]. Formally, let $N$ be a set of terminal nodes, with $|N|=n$. These nodes are located in a certain bounded convex region $S$ of the Euclidean space $R^{2}$. The problem instance is completely defined by the set of locations of $n$ terminal nodes, $N=\left\{x_{i} \leqslant S: 1 \leqslant i \leqslant n\right\}$, and the range assignment function RA. The resultant network topology will be an directed graph $G(N, E(N, R A))=G(N, R A)$, where $E(N, R A)$ is an edge set defined by $E(N, R A)=\left\{\left(x_{i}, x_{j}\right): x_{i}, x_{j} \leqslant N, i \neq j,\left\|x_{i}-x_{j}\right\| \leqslant R A\right\}$. A solution to the problem is a set of locations of relay nodes, $N_{R A}=\left\{y_{i} \leqslant S: 1 \leqslant i \leqslant N_{R A}\right\}$ and range assignment function $R A$ and represented in a single maximal connected component in the graph $G\left(N \cup N_{R A}, R A\right)$.

\section{Relevant work}

To our knowledge, the connectivity problem from this practical perspective has been discussed only in $[15,19,20]$. The MST algorithm proposed in [15] uses Boolean model for the network. The proposed scheme first calculates the MST and then placed the relay nodes on the edges of the MST that are longer than transmitting range $r$. The overall complexity of the algorithm is determined by the first step which is $O(N \log N)$. In [20], the problem was studied from network lifetime viewpoint. The paper discussed the problem how to increase the network lifetime by choosing the locations of relay sensors such that the Euclidean distances among sensors are at most some $\delta$ i.e. the range of the sensors. The algorithm computes the Steiner points and; then sorts the edges and placed relay sensors on the edges larger than the transmitting. The overall complexity of the algorithm is determined by the sorting step, which is $O(N \log N)$.

Kirousis et al. [14] showed that the range assignment problem for one-dimensional networks is in $P$ but NP-hard in the case of three-dimensional networks. For two-dimensional networks, the problem is remains NP-hard [5]. They also gave a minimum spanning tree (MST) based 2-approximation algorithm minimum power symmetric connectivity. In fact, their dynamic programming algorithm solved the complete range assignment problem in $O\left(n^{4}\right)$ for the case of $n$ collinear nodes and some power requirement. Recall, the goal of the complete range assignment problem is to establish a strong connected subgraph of a given graph. Calinescu et al. [3] improved the result [14] and proposed the fully polynomial $1+\ln 2$ approximation scheme base on the Steiner tree.

Blough et al. [1] consider a constrained version of the range assignment problem, where the node transmitting ranges must be assigned in such a way that the resulting communication graph is strongly connected and the energy cost is minimum. The result presented in this paper have shown that a weakly symmetric range can reduce the energy cost. Clementi et al. [4] presented the first polynomial-time, approximation algorithm for the Min Assignment problem. The algorithm guarantees a 2-approximation ratio and runs in $O\left(h n^{3}\right)$ time. The Min Assignment problem consists in finding a range assignment of minimum power consumption for a given set of radio stations located on a line and an integer $h \geqslant 1$. Rodoplu et al. [29] proposed a distributed algorithm to construct topology, which is guaranteed to contain 
the least energy path connecting any pair of nodes in the unit disk graph. Their algorithm relies on a simple radio propagation model for transmit power roll-off. Using this they achieved the minimum power topology, which contains the minimum-power path from each node to a designated node. However, their protocol is not time and space efficient. Li et al. [16] improved the result [29] by proposing an efficient localized algorithm to construct a topology that is sub-graph of the graph constructed by Rodoplu et al. [29] and proved that the sub-graph is sparse, i.e. it has a linear number of links.

Other researchers working in the field of wireless ad hoc and sensor networks have also consider the issue of power efficiency and transmitting range but have taken different approaches. For example, Hou et al. [12] approach was to adjust transmitting ranges of the given node to reduce overall consumption of the network. Particularly, they analyze the effect of adjusting transmission power to reduce interference and hence achieve higher throughput with respect to the fixed transmission ranges of the nodes [32]. On the other hand, Heinzelman et al. [11] approach was to assign the task of maintaining connectivity to energy nodes. They describe an adaptive clustering-based routing protocol that maximizes network lifetime by randomly rotating the role of per-cluster base station among nodes with higher energy reserves.

\section{Preliminaries}

This paper assumes that underline graph is geometric graph. A geometric graph $G=(V, E)$ is a graph in the plane so that its vertices are points and its edges are straight-line segments connecting pairs of these points. Recall, a geometric graph need not be planar. We represent sensors with points, relay sensors with additional points or Steiner points, and sensors transmission radius or distance between two sensors with edge length or Euclidean distance.

As we have mentioned above, a range assignment for the set $N$ on network nodes is a function RA that assign to $u \in N$ a transmitting range $R A(u)$. Formally, let $N$ be a set of nodes in the Euclidean space $R^{d}, d=2$. The problem is to determine a range assignment function RA such that corresponding communication graph is strongly connected and $\operatorname{Cost}(\mathrm{RA})=\sum_{u \in N}(R A(u))^{\alpha}$ is minimum over all connecting range assignment functions, where $\alpha$ is the distance-power gradient.

Let $N$ be a set of $n$ planar points. The Voronoi diagram partitions the plane into regions such that each region contains exactly one point. The region of a point $n_{i} \in N$ consists of all points closer to $n_{i}$ than to any other points on the plane. The region of a point $n_{i} \in N, 1 \leqslant i \leqslant n$, is called the Voronoi cell or Voronoi polygon and denoted as $V P\left(n_{i}\right)$. The vertices of these polygonal regions are called Voronoi vertices and the polygonal boundaries i.e., edges of the regions, are called Voronoi edges. The collection of Voronoi polygons $V P\left(n_{i}\right)$ for each $n_{i} \in V$ is called Voronoi diagram and often denoted as $V D(N)$. For further discussion on the properties of the Voronoi diagram, see [18].

The Delaunay triangulation, $D T(N)$, is the straight-line dual of Voronoi diagram $V D(N)$. Each Delaunay face (triangle) corresponds to a Voronoi vertex. The interior of each Delaunay triangle of $D T(N)$ contains no point $n_{i} \in N$. The Voronoi diagram and the Delaunay diagram can be constructed in $O(n \log (n)$ time [31]. Reader may consult [2].

Lemma 1. Given a set $N$ of $n \geqslant 3$ points. The $D T(N)$ has $E=3(n-1)-|\partial C H(N)|$ number of edges and $T=2(n-1)-|\partial C H(N)|$ number of triangles, where $|\partial C H(N)|$ is the number of points on the boundary of the Delaunay triangulation of the point set $N$.

Lemma 2. $D T(N)$ contains at least one MST for $N$. The MST for $N$ can be determined in time $O(n)$ from $\mathrm{DT}(\mathrm{N})[27]$. 


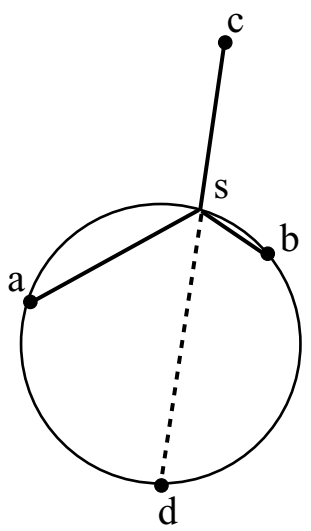

Fig. 1. The addition of Torricelli Point.

The Euclidean Steiner minimal tree (ESMT) problem asks for a shortest tree spanning a given set $N$ of $n$ terminals in the plane. Contrary to the minimum spanning tree (MST) problem, connections in Steiner minimum trees (SMTs) are not required to be between the terminals only. Additional intersections, called Steiner points, can be introduced to obtain shorter spanning tree. The ESMT problem is as follows. For a given set $N$ of points in the plane, construct a minimal length tree, which connects terminals in $N$. In order to minimize the total length of the tree, additional points $S=s_{1}, \ldots, s_{m}$ can be used. Here, the distance metric is the $L_{2}$ metric function, known as the Euclidean metric.

\subsection{Relevant Steiner tree properties}

We shall mention here only some basic properties of SMTs needed in the paper. The reader referred to [13] for a comprehensive survey. (1) Steiner points are incident with exactly three edges making $120^{\circ}$ with each other (angle condition). (2) SMTs for $n$ terminals have at most $n-2$ Steiner points. (3) SMTs are union of full Steiner trees (FSTs) terminals spanned by an FST have degree one. FSTs have two Steiner points less than they have terminals. If two FSTs share a terminal $N$, then the two edges incident make at least $120^{\circ}$ with each other. Therefore, no terminal can be in more than three FSTs. In other words, each terminal $N_{i}, 1 \leqslant i \leqslant n$, has at most degree 3 i.e., $\operatorname{deg}\left(n_{i}\right) \leqslant 3$. (4) It has been conjectured that the MST is no more than $\frac{2}{\sqrt{3}}$ times as long as the minimum length ESMT [9]. Polak has shown that this conjecture is true for $n=4$. Graham and Hwang [10] showed that the lower bound on the $\mathrm{SMT} / \mathrm{MST} \geqslant \frac{1}{\sqrt{3}}$. The problem has been shown to be NP-Complete in [8].

\subsection{Relevant Steiner tree results}

Now, we present some classical results regarding 3-terminal and 4-terminal Steiner tree problem and discuss their incorporation into the framework of our algorithms.

In case of three terminals, if one of the angles of $\triangle a b c$ is at least $120^{\circ}$, then the Steiner tree consists of simply the two edges subtending the obtuse angle. Therefore, assume that all internal angles of $\triangle a b c$ are less than $120^{\circ}$. The addition of an additional point (or Torricelli point) in $\triangle a b c$ is depicted in Fig. 1 [13].

Lemma 3. An additional point in the plane, the sum of whose distances from three given points is minimal, can be located if and only if all internal angles of a triangle are less than $120^{\circ}$. 


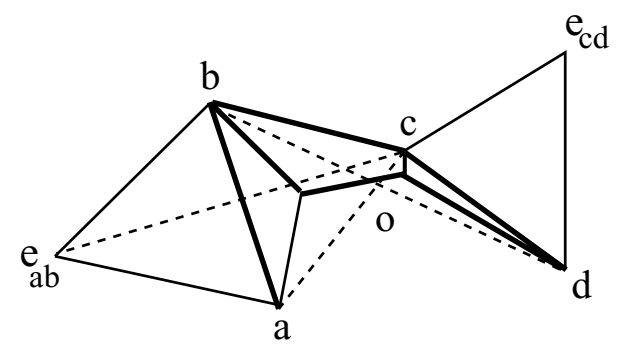

Fig. 2. The Quadrilateral abcd.

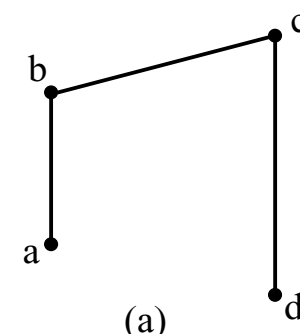

(a)

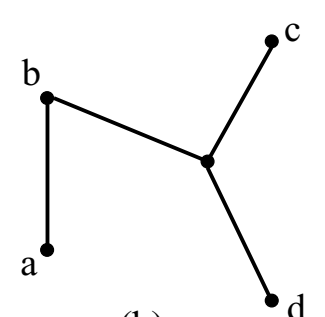

(b)

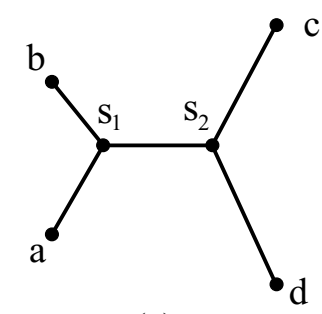

(c)

Fig. 3. Illustrates the addition of 2 additional points in quadrilateral. For proof of the fact that (a), (b), and (c) in the Figure are sufficient to define the optimal Steiner tree for 4 terminals see Gilbert and Polak [14].

In case of four terminals, consider the quadrilateral abcd in the Fig. 2. Points $e_{a b}$ and $e_{c d}$ form equilateral triangles with $(a b)$ and $(c d)$ respectively. By Du et al. [7] the necessary and sufficient condition for the existence of FST in that the quadrilateral abcd must be convex. Weng [34] showed that convexity of the quadrilateral abcd (see Fig. 2) is implied by following two conditions.

1. $\angle e d a a b, \angle e a b c d, \angle e d a a b$, and $\angle e a b c a$ are all less than $120^{\circ}$.

2. $\angle d o c<120^{\circ}$.

An important insight of Polak's [26] paper on existence of SMT of four terminals can be stated as follows.

Lemma 4. If four terminals $a, b, c$, and $d$ form a convex quadrilateral, then SMT exists for those terminals.

\section{Computational model}

The communication graph defines the set of wireless links that the nodes can use to communicate with each other. Based on the above discussion, the existence of the edge (link) between two nodes depends on the distance between the nodes, transmission range assignment, and environmental conditions i.e., distance power gradient, $\alpha$. Given a transmitting range assignment, we define communication graph $G$ such that directed edge $(u, v) \in G$ if, and only if, $\operatorname{dist}(u, v) \leqslant r(u)$ i.e., Euclidean distance from $u$ to $v$ is at most $r(u)$.

This paper assumes the long-distance model [28], which abstracts many characteristics of the environment. In this model, the average long-distance path loss is proportional to the separation distance 
between transmitting node $v$ and receiving node $\mathrm{t}$ raised to a certain exponent, $\alpha$, which is called the distance power-gradient. The value of $\alpha$ depends on the environmental conditions and it has been experimentally evaluated in many scenarios [30]. Typically, nodes are located in a two-dimensional Euclidean space. These nodes are connected by wireless links that are induced by their energy level. According to the long-distance model, in the given region, each node $u$ transmits a signal with power $P(u)$, which is received by other node $v$ such that $\operatorname{dist}(u, v)^{\alpha} \leqslant P(u) / \gamma$, where $\operatorname{dist}(u, v)$ is the Euclidean distance between nodes $u$ and $v, \alpha \leqslant 1$ is the distance power gradient, and $\gamma \leqslant 1$ is the transmission quality parameter. Without loss of generality, in this paper, we assume $\gamma=1$. The radio coverage region in this model is a disk of radius at most $r(u) \geqslant(P(u) / \gamma)^{1 / \alpha}$ centered at the transmitter $u$. Since $\gamma=1$, thus we have $r(u) \geqslant(P(u))^{1 / \gamma}$. The value $r(u)$ is the transmission range of transmitter $u$, i.e., the maximum distance at which $u$ can transmit in one hop with power $P(u)$.

\section{Overall strategy of the algorithm}

For the ease of the presentation, we divide the overall algorithm's strategy into four distinct phases namely, construction phase, test phase, merge phase, and the reduction phase. The preliminary version (Kruskal's version) of this work appeared in the Reference [24]. Our algorithm operates much like Prim's algorithm for finding the minimum spanning tree. This algorithm has the property that the adding of edges in the set always forms a single tree [27]. As illustrated in the example below, the algorithm starts by placing terminal in the priority queue. The tree starts from an arbitrary terminal and grows from there until the tree spans all the vertices in $N$. At every stage, it selects the shortest available edge that can extend the tree to an additional terminal and mark the terminals up to 4-terminals for which edges are making right turns or left turns. The algorithm ends the construction phase by partitioning the set into subsets of 4-, 3- and 2-terminals using markings. Note that since we are using right turn (left turn) technique to create a subset, at least one terminal is common in adjacent subsets. The performance of this phase depends on how we implement the priority queue. If queue is implemented as a binary heap, building heap requires $O(n)$ time. Since each operation that requires to extract the minimum runs $O(n)$ time, and there are $O(n)$ such operations therefore, the total time is $O(n \log n)$. Selecting the shortest edge from the neighboring edges requires $O(n)$ time. Thus, the total time for construction phase is $O(n \log n)$. However, the asymptotic running time of this phase can be improved by implementing priority differently e.g. Fibonacci heap.

In the test phase, the algorithm first performs the convexity test using lemmas 1 and 2 for 3 -terminal subset and 4-terminal subset. After that, it performs the length test to find out if there is any edge in the subsets whose length is greater than $\delta>0$. If such an edge exists in 3-terminal and 4-terminal subsets, additional points (relay sensors) are required to increase the lifetime of network. Subsets that pass the convexity test are those whose overall lengths can be minimize by adding Steiner points. Hence, increase the lifetime of network. The algorithm includes the additional point in 3-terminal subsets using the algorithm in [33] (or see Fig. 1) and additional two points in 4-terminal subsets using simple geometry in [17] (or see Lemma 4 or Fig. 3). It is easy to see that operations in this phase require constant time. Therefore, this phase require linear time.

In the merging phase, the algorithm simply combines the 2-terminal, 3-terminal and 4-terminal subset by union operation. Since at least one terminal is common in adjacent subsets, union operation will not create the cycles.

Lastly, the reduction phase computes the lengths of each edge in the tree produced by merging phase in $O(n)$ time. Sort the edges in non-decreasing order of lengths using some optimal sorting algorithm 
(e.g. heap sort) that require $O(n \log n)$ time. For all edges, if the length of edge is greater than $\delta$ then add $\left(\left\lceil\frac{\left|e_{i} e_{i+1}\right|}{\delta}\right\rceil-1\right)$ 2-degree points which can be done in $O(n)$ time. Thus, the total time for reduction phase is $O(n \log n)$.

The output of the algorithm is the tree that spans all the terminals of the given set $N$ whose each edge has a length at most given positive $\delta$.

\subsection{Algorithm ADD NODE}

In this section, we formalize the idea presented in the preceding section. The goal of our algorithm is to abandon long-distance edges (communication links) and establish small edges instead. For this purpose, the algorithm chooses a location of additional nodes or relay node based on the Steiner topology. The following algorithm is based on the Prim's algorithm appeared in [20] in the context of energy conservation in wireless networks and the version based on the Kruskal's algorithm appeared in [24].

1. Given a set $N$ of $n$ terminals. Place $n_{i} \in N$ in a priority queue. Call this priority queue $Q$.

2. For each $n_{i} \in Q$, select the shortest available edge in $\operatorname{Adj}\left[n_{i}\right]$.

3. Check whether new edge making right turn (left turn) with previous edge.

4. Mark all terminals (up to 4 terminals) corresponding to consecutive edges that are making right turns (left turns). At the end of this step, we will have connected sub-graphs consisting of 4-, 3-, and 2-terminals.

5. Check the convexity of the 3 - and 4-terminal subsets using Lemmas 3 and 4 respectively and include additional points if sub-graphs pass these tests.

6. Merge 2-, 3-, and 4-terminal subsets (sub-graphs).

7. Suppose $e_{1}, e_{2}, \ldots, e_{n-1}$ are all edges in the resultant tree. Compute $l\left(e_{i}\right)$ for each edge $e_{i}$.

8. Sort the edges $e_{i}, 1 \leqslant i \leqslant n-1$, in a non-decreasing order of $l($.$) .$

9. For all $n-1$ edges, If $\left|e_{i} e_{i+1}\right|>\delta$, add $\left(\left\lceil\frac{\left|e_{i} e_{i+1}\right|}{\delta} \mid-1\right)\right.$, where $1 \leqslant i \leqslant n-1,2$-degree additional points.

\subsection{Correctness}

In this section, we shall show that the resultant graph produced by the algorithm is a minimum length tree. Following lemma is based on $[19,20]$.

Lemma 5. The tree $\mathrm{T}$ produced by the algorithm is minimal if and only if each $(u, v) \notin T$ and for all edges $(x, y)$ in the cyclic graph arising from $\mathrm{T}$ by adding $(u, v), C_{T}(u, v)$, we have $l(u, v) \geqslant l(x, y)$, where $l(u, v)$ denotes the Euclidean distance (edge length) between $u$ and $v$.

Proof: Suppose that the tree $T$ produced by the algorithm is minimal. If $l(u, v)<l(x, y)$ (i.e., condition given in the Lemma 3 does not satisfy) then there must exist an edge $(u, v) \notin T$ and an edge $(x, y) \in C_{T}(u, v)$ with $l(u, v)<l(x, y)$. Since edge $(x, y)$ is a bridge edge, removed of $(x, y)$ from $T$ divides $T$ into two components. Adding $(u, v)$ i.e., $(u, v) \cup T-\{x, y\}$ gives a new tree $T^{\prime}$. Since $l(u, v)<l(x, y), T^{\prime}$ has smaller length than $T$. This contradicts the minimality of $T$.

Conversely if $l(u, v) \leqslant l(x, y)$. Suppose algorithm produced a tree $T$ which spans given set of points and show that some minimal spanning tree $T^{\prime}$ produced by same set of points is same as that of $T$ in terms of length i.e., $l(T)=l\left(T^{\prime}\right)$. This will prove that $T$ is minimal as well. We use induction on the edge $m \in T^{\prime}-T$. When $m=0, T=T^{\prime}$ is trivial. So let $\left(u^{\prime}, v^{\prime}\right) \in T^{\prime}-T$, If we remove $\left(u^{\prime}, v^{\prime}\right)$ from 
$T^{\prime}$, the $T^{\prime}$ is divided in two connected components $C_{1}$ and $C_{2}$. If we add the path $C_{T}\left(u^{\prime}, v^{\prime}\right)-\left\{u^{\prime}, v^{\prime}\right\}$ to $T^{\prime}-\left\{u^{\prime}, v^{\prime}\right\}$ i.e., $T^{\prime}-\left\{u^{\prime}, v^{\prime}\right\} \cup C_{T}\left(u^{\prime}, v^{\prime}\right)-\left\{u^{\prime}, v^{\prime}\right\}$, connected components $C_{1}$ and $C_{2}$ becomes connected again. Therefore, the graph arising from $T$ by adding $\left(u^{\prime}, v^{\prime}\right), C_{T}\left(u, v^{\prime}\right)$, has to contain an edge of $T^{\prime}$, because otherwise $T^{\prime}-\left\{u^{\prime}, v^{\prime}\right\}$ would still be connected. The minimality of $T^{\prime}$ implies that $l(u, v) \geqslant l\left(u^{\prime}, v^{\prime}\right)$ : replacing edge $\left(u^{\prime}, v^{\prime}\right)$ by $(u, v)$ in $T^{\prime}$, we get another tree $T^{\prime \prime}$ and if $l(u, v)<l\left(u^{\prime}, v^{\prime}\right)$, this tree would have smaller length than $T^{\prime}$ contradicting the minimality of $T^{\prime} T$. On the other hand, lemma states that $l\left(u^{\prime}, v^{\prime}\right) \geqslant l(u, v)$, so that $l\left(u^{\prime}, v^{\prime}\right)=l(u, v)$ and $l\left(T^{\prime \prime}\right)=w\left(T^{\prime}\right)$. Therefore, $T^{\prime \prime}$ is a minimal spanning tree as well. $T^{\prime \prime}$ has one more edge in common with $T$ than $T^{\prime}$, and using induction, we have $l(T)=l\left(T^{\prime \prime}\right)=l\left(T^{\prime}\right)$. This completes the proof.

\section{Approximations for range assignment}

In this section, we present an approximation of the optimal solution of range assignment problem by using the algorithm ADD_NODE. In essence, we are defining the range assignment, RA, on the tree produced by the ADD_NODE algorithm.

\section{Approximation}

\section{ADD_NODE}

2. For each node $u \in T$, define range assignment, $R A_{t}=\max _{(u, v) \in T} \operatorname{dist}(u, v)$.

Now we show that the above "approximation algorithm" produces a 2-approximation of the optimal solution. The following lemma is based on [14].

Lemma 6. Let $R A_{O p t}$ be an optimal range assignment of the problem and $R A_{T}$ be the range assignment defined above [see line 2 of the Approximation]. Then $\operatorname{Cost}\left(R A_{T}\right)<2 \times \operatorname{Cost}\left(R A_{\text {opt }}\right)$

Proof: For the proof, we use the technique described in [30]. It consists of two steps. First, we show that $\operatorname{Cost}\left(R A_{\text {opt }}\right)$ is greater than the cost of tree produced by the algorithm i.e., $\operatorname{Cost}(\mathrm{T})$. Then, we show that cost of range assignment on the resultant tree, $\operatorname{Cost}\left(R A_{T}\right)$ is less than twice of the cost of resultant tree i.e., $\operatorname{Cost}\left(R A_{T}\right)<2 \operatorname{Cost}(\mathrm{T})$. Firstly, $\operatorname{Cost}\left(R A_{\text {opt }}\right)>\operatorname{Cost}(\mathrm{T})$ : For optimal assignment a given set $\mathrm{N}$ of $\mathrm{n}$ points, construct a shortest path destination tree rooted at $u$. Given the shortest path destination tree, the correspond tree $T^{\prime}$ (say) can changing the directed edges. Since each of the $n-1$ nodes other than the root must be assigned a range, we have $\operatorname{Cost}\left(R A_{\text {opt }}\right)>\operatorname{Cost}\left(T^{\prime}\right)$. Secondly, since each edge of the resultant tree $T$ can be chosen at most by two nodes, we have $\operatorname{Cost}\left(R A_{\text {opt }}\right)<2 \times \operatorname{Cost}(T)$.

Now we show that the Approximation algorithm satisfies the strong connectivity property (Proposition 2) i.e. the resultant graph (tree) produced by the Approximation is strongly connected.

For this purpose, we define the minimal distance between $u$ and $v$ over all nodes as follows: $\operatorname{dist}_{u}(N)=$ $\min \{\operatorname{dist}(u, v): u \in N$ and $v \in N \backslash\{u\}\}$. Note that this idea is depend upon the notion of well-spread defined in [6]. We claim that for a given set of nodes $N$, a range assignment $R A$ in the approximation, the condition $R A(u) \geqslant \operatorname{dist}_{u}(N)$ must hold for all nodes in the set $N$. We formalize our claim as follows: 
Lemma 7. For a given set of nodes $\mathrm{N}$, if a range assignment $\mathrm{RA}$ allocated by the Approximation is strongly connected, then $R A(u) \geqslant \operatorname{dist}_{u}(N)$ for all $u \in N$.

Proof: Assume to the contrary, that there exists a range assignment $R A$ in the Approximation such that $R A(u)<\operatorname{dist}_{u}(N)$. That is, condition given in line 2 of the Approximation algorithm does not hold for some particular but arbitrarily chosen nodes $u$ and $v$. Then, by the Proposition 1, if there was a node $u$ with $R A(u)<\operatorname{dist}_{u}(N)$, it could not send data to any other node (terminal or relay node). This implies that there cannot be an edge (communication link) leaving the node $u$. Thus, $G_{R A}$ cannot be connected. Since $G_{R A}$ is not connected therefore, it cannot be strongly connected. This contradicts the supposition that $R A(u)<\operatorname{dist}_{u}(N)$. Hence, the supposition is false and the lemma is true. This completes the proof.

Indeed, the same node connectivity adopted in the Lemma 7 can be use to prove the following stronger result about the connectivity in the resultant graph produced by the algorithm ADD NODE. In particular, the following lemma shows that $G_{R A}$ preserves the connectivity of $G_{A D D \_N O D E}$.

Lemma 8. The resultant graph, $G_{R A}$, produced by the approximation algorithm is connected if, and only if, the graph $G_{A D D \_N O D E}$ produced by the algorithm ADD_NODE is connected.

Proof: For necessary condition, suppose $u$ and $v$ are any particular but arbitrarily chosen nodes in $G_{R A}$. Then by line 2 of the approximation algorithm, the algorithm will consider only those edges that are connected in the graph $G_{A D D_{-} N O D E}$. Therefore, nodes $\mathrm{u}$ and $\mathrm{v}$ are connected and this is what to be shown.

For sufficient condition, assume to the contrary, that there exists at least one edge in $G_{A D D \_N O D E}$ that is connected i.e. $\operatorname{edge}(u, v) \in G_{A D D_{-} N O D E}$ and not connected in $G_{R A}$ i.e., edge $(u, v) \notin G_{R A}$. Consider the edge $(u, v)$ with minimum range assignment i.e., Euclidean distance $\operatorname{dist}(u, v)$, among

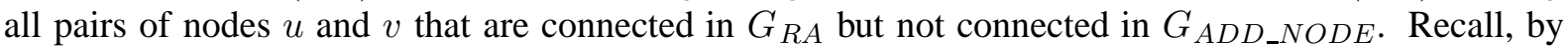
definition edge $(u, v) \in E_{R A}$ iff $R(u) \geqslant \operatorname{dist}(u, v)$ so, minimum range assignment means the edge connecting node $u$ and node $v$ in the $G_{A D D \_N O D E}$. The node $u$ and $v$ must be connected directly by edge $(u, v)$ in $G_{A D D \_N O D E}$ (see line 2 of ADD_NODE algorithm). Otherwise, a different edge $(x, y)$ must exist on the path connecting nodes $u$ and $v$ would have a Euclidean distance (range assignment) $\operatorname{dist}(x, y)$ less than $\operatorname{dist}(u, v)$ and nodes $u$ and $v$ would not be the edge with minimum range assignment i.e. minimum Euclidean distance, $\operatorname{dist}(u, v)$. Since the edge $(u, v)$ is in $G_{A D D} N O D E$ by line 2 of the algorithm ADD_NODE, the cost of the shortest path connecting nodes $u$ and $v$ is their Euclidean distance i.e. $R A=\operatorname{dist}(u, v)$. Since $\operatorname{dist}(u, x)$ is less than $\operatorname{dist}(u, v)$. Also, since $\operatorname{dist}(u, v)$ is the least such value for any non-connected nodes in $G_{R A}$ and $u$ and $x$ must also be connected in $G_{R A}$ (by line 2 in approximation algorithm). For the same reason, node $v$ and node $x$ must be connected in $G_{R A}$. This contradicts the supposition that nodes $u$ and $v$ are not connected in the graph $G_{R A}$ produced by algorithm approximation. Hence, the supposition is false and the sufficient condition holds. And this completes the proof.

\section{Open question}

The goal of our proposed scheme is to discard long-distance communication links and instead create small links (range assignments). For this purpose, the algorithm chooses a location of additional nodes or relay node using the notion of Steiner tree construction. Clearly, we cannot throw away nodes that 
are too far from neighbor nodes otherwise, the resultant graph will be partitioned. In general, there is a trade-off between network connectivity and sparseness. Recall, sparseness means that the number of edges in the graph should be in the order of the number of nodes. Although the graph constructed by the approximation algorithm has a nice feature of being at most twice the cost of an optimal cost while maintaining the connectivity, the question of whether the constructed graph is actually a sparse remains open. Despite of the observation that there exists a node placement and transmitting range setting such that some long-distance links can be removed from the resultant communication graph without increasing the cost of the algorithm. We strongly suspect that answer to the question is negative (at least in the context of our problem setting). Our argument is as follows. There always exists a node configuration and transmitting range setting such that the number of communication links in the graph constructed by ADD_NODE algorithm equals to the number of links in the resultant graph.

\section{Conclusion}

The paper discussed the algorithmic issues concerning the problem of introducing additional nodes (relay nodes) in the existing wireless network from practical viewpoint. That is, setup a communication links in the emergencies by introducing additional or relay nodes. The paper also presented a 2approximation algorithm to assign the transmitting ranges in such a way that the resultant network is connected.

\section{References}

[1] D.M. Blough, M. Leoncini, G. Resta and P. Santi, On the Symmetric Range Assignment Problem in Wireless Ad Hoc Networks, in Proceedings of 2nd IFIP International Conference on Theoretical Computer Science: Foundations of Information Technology in the Era of Networking and Mobile Computing, pp. 71-82, 2002.

[2] B. Boots, A. Okabe, K. Sugihara and S. Chiu, Spatial Tessellations: Concepts and Applications of Voronoi Diagrams, Wiley, New York, 2000.

[3] G. Calinescu, I. Mandoiu and A. Zelkovsky, Symmetric connectivity with minimum power consumption in radio networks, in Proceedings of 2nd IFIP International Conference on Theoretical Computer Science: Foundations of Information Technology in the Era of Networking and Mobile Computing, pp. 119-130, 2002.

[4] A.E.F. Clementi, A. Ferreira, P. Penna, S. Perennes and R. Silvestri, The mimimum range assignment problem on linear radio networks, Algorithmica 35(2)(2003), 95-110.

[5] A.E.F. Clementi, P. Penna and R. Silvestri, Hardness Results for the Power Range Assignment Problem in Packet Radio networks, in Proceedings of 2nd Workshop on Approximation algorithms for Combinatorial Optimization Problems (RANDOM/APPROX'99), pp. 197-208, 1999.

[6] A.E.F. Clementi, P. Penna and R. Silvestri, On the power assignment problem in radio networks, Mobile Networks and Applications 9(2) (2004), 125-140.

[7] D. Du, F. Hwang, G. Song and G. Ting, Steiner minimal trees on sets of four points, Discrete Computational Geometry 2(1) (1987), 401-414.

[8] M.R. Garey, R.L. Graham and D.S. Johnson, The complexity of computing Steiner minimal trees, SIAM Jornal on Applied Mathematics 32(4) (1977), 835-859.

[9] E.N. Gilbert and H.O. Polak, Steiner minimal trees, SIAM Journal on Applied Mathematics 1(16) (1968), 1-29.

[10] R.L. Graham and F.K. Hwang, Remarks on Steiner minimal trees, Bulletin of the Institute of Mathematics Academia Sinica 4(1) (1976), 177-182.

[11] W.R. Heinzelman, A. Chandrakasan and H. Balakrishnan, Energy-Efficient Communication Protocol for Wireless MicroSensor networks, in Proceedings of IEEE Hawaii International Conference on System Sciences, pp. 4-7, 2000.

[12] T.C. Hou and V.O.K. Li, Transmitting range control in multihop packet radio networks, IEEE Transactions on Communications 34(1) (1986), 38-44.

[13] F. Hwang, D. Richards and P. Winter, The Steiner tree problem, Annals of Discrete Mathematics 53, Elsevier, Amsterdam, 1992. 
[14] L.M. Kirousis, E. Krankis, D. Krizanc and A. Pelc, Power consumption in packet radio, Theoretical Computer Science 243(1-2) (2000), 289-305.

[15] H. Koskinen, J. Karvo and O. Apilo, On Improving Connectivity of Static Ad-Hoc Networks By Adding Nodes, In Challenges in Ad Hoc Networking, pp. 169-178. Springer, Boston, 2006.

[16] X.Y. Li and P.J. Wan, Constructing Minimum Energy Mobile Wireless Networks, in Proceedings of the 2nd ACM International Symposium on Mobile Ad Hoc Networking and Computing (MobiHoc 2001), pp. 283-286, 2001.

[17] Z.A. Melzak, Companion to Concrete Mathematics, Wiley, New York, 1973.

[18] R.B. Muhammad, A parallel Algorithm for Planar Voronoi Diagram on Hypercube Model of Computation, in Proceedings of the 16th IASTED International Conference on Parallel and Distributed Computing and Systems (PDCS'04), Cambridge, Massachusetts, USA, pp. 542-547, 2004.

[19] R.B. Muhammad, Connectivity Setup using Steiner Tree in Ad Hoc Wireless Networks, in Proceedings of Eight Annual IEEE Wireless and Microwave Technology Conference (WAMICON'06), Clear Water, Florida, USA, pp. five pages, full text on CD-ROM, 2006.

[20] R.B. Muhammad, Deterministic Energy Conserving Algorithms for Wireless Sensor Networks, in Proceedings of the IEEE International Conference on Networking Sensing and Control (ICNSC'06), Ft. Lauderdale, Florida, USA, pp. 324-329, 2006.

[21] R.B. Muhammad, A Parallel Local Search Algorithm for Euclidean Steiner Tree Problem, in IEEE Proceedings of the 7th International Conference on Software Engineering, Artificial Intelligence Networking and Parallel/Distributed Computing (SNPD'06), Las Vegas, USA, pp. 157-164, 2006.

[22] R.B. Muhammad, Parallelization of Local Search for Euclidean Steiner Tree Problem, in Proceedings of The 44th ACM Southeast Conference (ACMSE'06), Melbourne, Florida, USA, pp. 233-238, 2006.

[23] R.B. Muhammad, Range Assignment Approximation Based on Steiner Tree in Ad Hoc Wireless Networks, in Proceedings of 22nd IEEE International Conference on Advanced Information Networking and Applications (AINA'08), Okinawa, Japan, pp. 100-105, 2008.

[24] R.B. Muhammad, Transmitting Range Assignment using Steiner Tree in Ad Hoc Wireless Networks, in IEEE Proceedings of the 5th International Conference on Information Technology: New Generations (ITNG'08), Las Vegas, USA, pp. 408413, 2008 .

[25] K. Pahlavan and A. Levesque, Wireless Information Networks, Wiley-Interscience, New York, 1995.

[26] H.O. Polak, Some remarks on the Steiner problem, Journal of Combinatorial Theory 24(3) (1978), 278-295.

[27] F.P. Preparata and M.I. Shamos, Computational Geometry: An Introduction, Springer-Verlag, New York, 1988.

[28] T. Rappaport, Wireless Communications: Principle and Practice, Prentice Hall, New Jersey, 2002.

[29] V. Rodoplu and T. Meng, Minimum energy mobile wireless networks, IEEE Journal on Selected Areas in Communications 17(8) (1999), 1333-1344.

[30] P. Santi, Topology Control in Wireless Ad Hoc and Sensor Networks, Wiley, Hoboken, NJ, 2005.

[31] M.I. Shamos, Geometric Complexity, in Proceedings of the 7th Annual ACM Symposium on Theory of Computing, pp. 224-223, 1975.

[32] H. Takagi and L. Kleinrock, Optimal transmission ranges for randomly distributed packet radio terminals, IEEE Transactions on Communications 23(3) (1984), 246-257.

[33] E.A. Thomson, The method of minimum evolution, Annals of Human Genetics London 36 (1973), 333-340.

[34] J. Weng, Restudy of Steiner minimal trees on four points, Preprint, 1991.

Rashid Bin Muhammad was born in Karachi, Pakistan. He is currently a Ph.D. candidate and a teaching fellow of computer science at Kent State University. He received his MS degrees in physics, industrial education, and computer science from University of Karachi (1990), Murray State University (1994), and Kent State University (2003), respectively. He is a member of both ACM and the IEEE Computer Society. His research interests include parallel and distributed computing, computational geometry, graph theory, and algorithmic issues in wireless networking. 

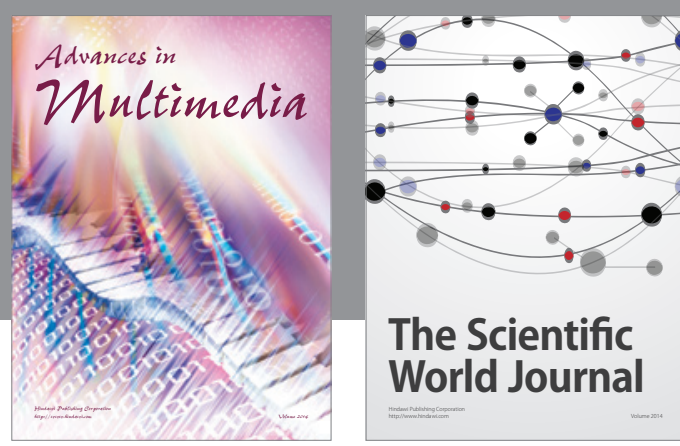

The Scientific World Journal
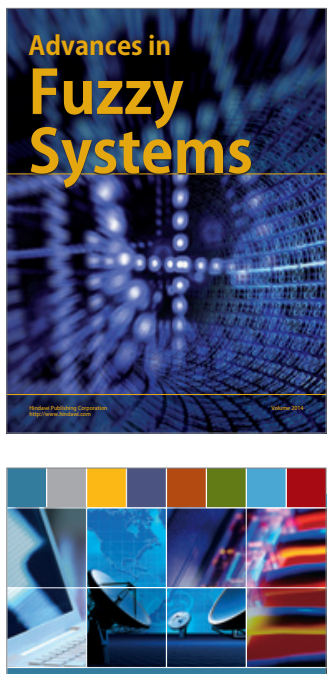

Computer Networks and Communications
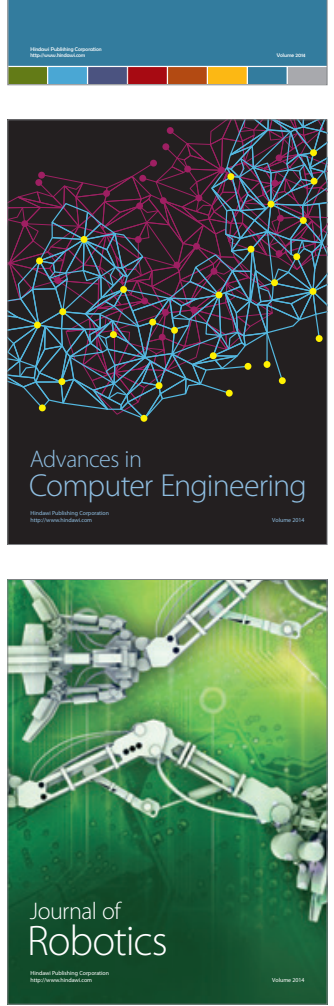
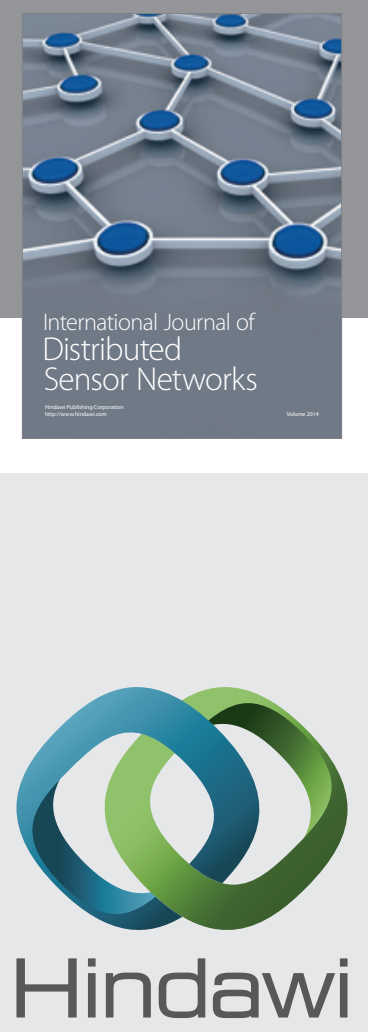

Submit your manuscripts at

http://www.hindawi.com
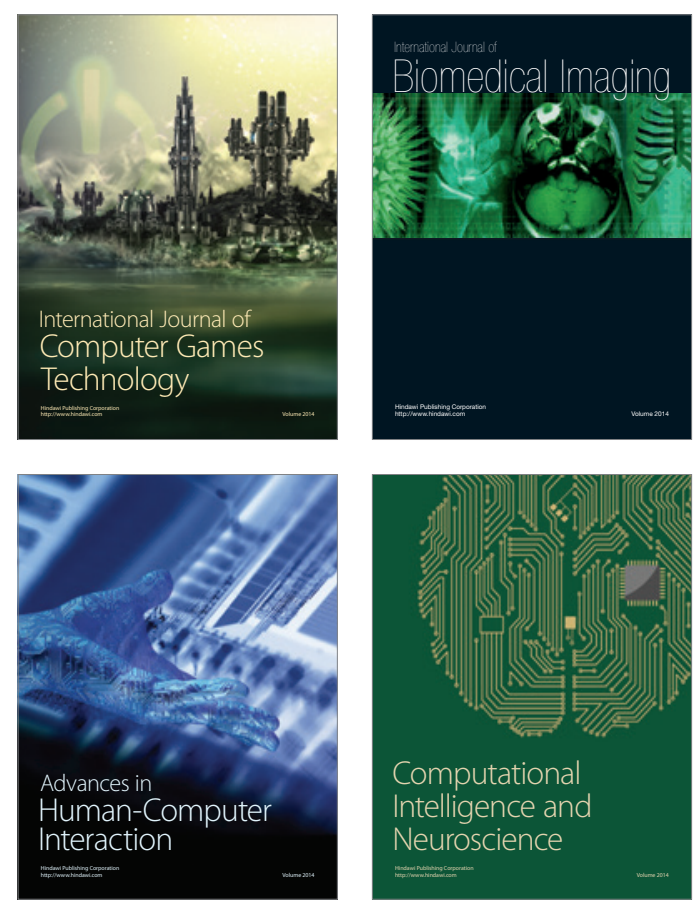
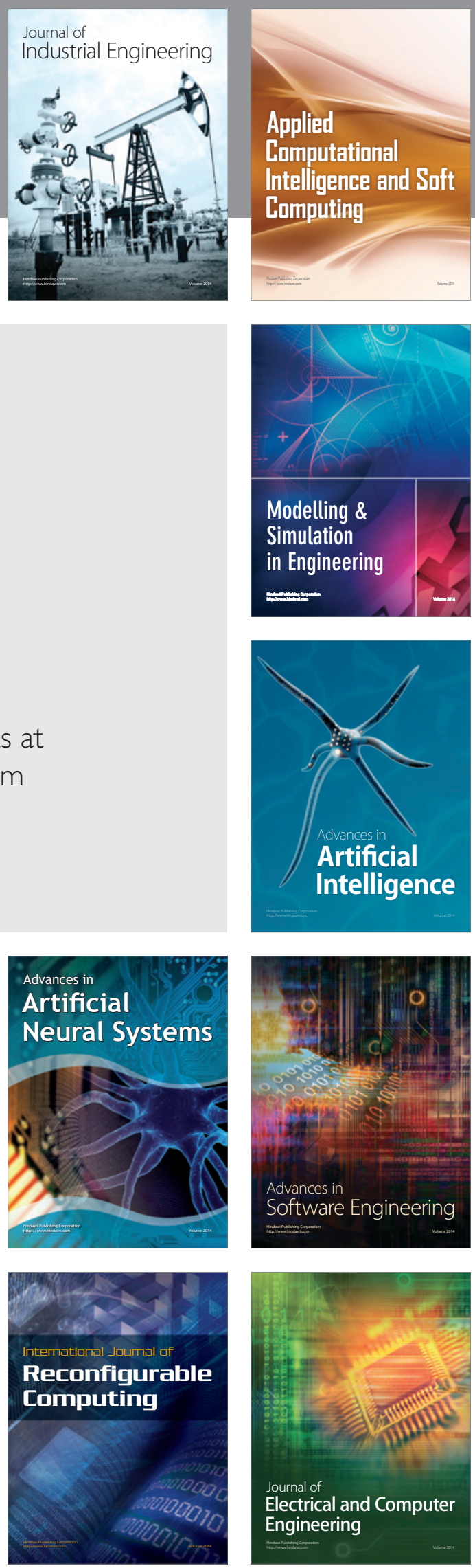